\title{
Marchenko Imaging: A least-squares approach implemented in Julia programming
}

\author{
Rafael A. de Paula*1, Daniel E. Revelo ${ }^{12}$, Reynam C. Pestana ${ }^{2}$, Diego F. Barrera ${ }^{1}$ \\ 1 SENAI/CIMATEC Supercomputing Center, Salvador, Bahia, Brazil \\ ${ }^{2}$ Federal University of Bahia (UFBA), Salvador, Bahia, Brazil
}

Copyright 2021, SBGf - Sociedade Brasileira de Geofísica

This paper was prepared for presentation at the $17^{\text {th }}$ International Congress of the Brazilian Geophysical Society, held in Rio de Janeiro, Brazil, 8 to 11 November, 2021.

Contents of this paper were reviewed by the Technical Committee of the $17^{\text {th }}$ International Congress of the Brazilian Geophysical Society and do not necessarily represent any position of the SBGf, its officers or members. Electronic reproduction or storage of any part of this paper for commercial purposes without the written consent of The Brazilian Geophysical Society is prohibited.

\begin{abstract}
Reverse-time migration (RTM) is the method commonly employed in seismic processing to produce depth images. However, it requires that the data to be migrated should be free of multiple-scattering events as surface-multiples and internal multiples. More recently, Marchenko imaging has been used as an efficient procedure that can efficiently remove these multiples events during the imaging process. The multiples events that appear in the RTM images as false events are completed eliminated during the Marchenko-redatuming step and thus the images produced by Green's functions are free of these artifacts. In this work, we discuss the solution of the coupled Marchenko equations using a least-squares (LS) approach and its implementation using the Julia programming language. The inverted focusing functions are then employed to obtain Green's functions and after that, a cross-correlation image condition is applied to generate a depth image. We test our LS-Marchenko method implemented in Julia using a simple geological model. Finally, the numerical results of the Marchenko image and RTM image are compared, validating the codes implemented in Julia.
\end{abstract}

\section{INTRODUCTION}

Seismic imaging is still a key step in seismic processing and the generation of seismic images can be done using different methods, such as Kirchhoff migration, reversetime migration (RTM), and more recently by Marchenko imaging (Baysal et al., 1983; Hokstad, 2000; van der Neut et al., 2015c; Wapenaar et al., 2014). Behind each methodology, there is a largely complex development of an algorithm to generate these images. Despite the existence of all these methodologies, the search for better imaging improvements continues today, and the understanding of how these techniques are implemented is necessary.

Marchenko imaging recently has become noticed because it can generate a high-quality image, despite its high computational cost. The Marchenko algorithm is a robust method that computes subsurface-to-surface Green's function, and its main advantage is that it can correctly treat the internal multiple reflections (Broggini and Snieder, 2012). Recent studies show that it is possible to calculate the Green's function for a synthetic source located at any subsurface position, which can be done by an iterative substitution scheme (van der Neut et al., 2015a; Thorbecke et al., 2017) or by a least-squares inversion algorithm (van der Neut et al., 2015a; Dukalski and de Vos, 2018).

Some works/libraries already have implementations of the Marchenko algorithm using high-level and lowlevel language interpretations (Ravasi and Vasconcelos, 2020b,a; Thorbecke et al., 2017). Thorbecke et al. (2017) present an overview of the Marchenko method using an iterative substitution scheme, and how to implement it. But there is no work showing how to do it for the leastsquares inversion scheme, because in some cases we cannot expand equations into a Neumann series (Ravasi, 2017; Vargas et al., 2021). In this specific situation, e.g., in free-surface case, it is not possible to retrieve the focusing function by iterative substitution, therefore, an inversion method is required, as well as understanding how to use the least-square scheme in the Marchenko method.

Nowadays, there are several programming languages and each one brings a different kind of advantage, e.g., better performance or simple syntax. Here, we choose to use the Julia programming language, which tries to unite both advantages. Julia, despite being a young language, has been efficient in both computation speed and easy code implementation (Bezanson et al., 2012). Julia was released in 2012 since then a lot of upgrades have been done. Also, the Julia language implements a lot of high-level features from interpreting languages. Due to this, the development of software on Julia is potentially faster (Gevorkyan et al., 2019).

This work provides a simple guide to applying the leastsquare (LS) approach in the Marchenko method using Julia programming language, but the logical programming can be done in any other, high or low, level interpreting languages. To show the efficiency of the method and the algorithm, we use a synthetic model with constant velocity and variable density to retrieve Green's functions and build the seismic image.

\section{THEORY}

\section{Marchenko equations}

We present a brief review of the Marchenko equations presented in Ravasi (2017). From reciprocity theorems of correlation and convolution types between two states (de Hoop, 1995; Wapenaar and Grimberg, 1996; Slob et al., 2014) and based on inverse scattering theory (Broggini and Snieder, 2012), it is possible to relate wavefields that focus the energy on a specific focal point in the subsurface to Green's function relative to this point that is recognized as a virtual source. These wavefields 
are known as focusing functions and the relation to up- and downgoing Green's functions $\left(g^{-}\right.$and $\left.g^{+}\right)$at the selected point in the subsurface can be written in the frequency domain as (Wapenaar et al., 2014)

$$
\begin{aligned}
& g^{-}\left(\mathbf{x}_{F}, \mathbf{x}_{R}\right)=\int_{\Lambda_{R}} R\left(\mathbf{x}_{R}, \mathbf{x}_{R}^{\prime}\right) f^{+}\left(\mathbf{x}_{R}^{\prime}, \mathbf{x}_{F}\right) d \mathbf{x}_{R}^{\prime}-f^{-}\left(\mathbf{x}_{R}, \mathbf{x}_{F}\right), \\
& -g^{+*}\left(\mathbf{x}_{F}, \mathbf{x}_{R}\right)=\int_{\Lambda_{R}} R^{*}\left(\mathbf{x}_{R}, \mathbf{x}_{R}^{\prime}\right) f^{-}\left(\mathbf{x}_{R}^{\prime}, \mathbf{x}_{F}\right) d \mathbf{x}_{R}^{\prime}-f^{+}\left(\mathbf{x}_{R}, \mathbf{x}_{F}\right),
\end{aligned}
$$

where $\Lambda_{R}$ represents the acquisition level, $\mathbf{x}_{R}^{\prime}$ is the source position at the level $\Lambda_{R}$ and $R$ is the reflection response from $\mathbf{x}_{R}^{\prime}$ to the receiver at $\mathbf{x}_{R} . f^{-}$and $f^{+}$are the up- and downgoing focusing functions, respectively. The superscript $*$ denotes the complex conjugate of the wavefield (i.e., a time reversal in the time domain) and the integrals in both equations correspond to multidimensional space-frequency convolution and correlation, respectively. Equations 1 and 2 are named as the coupled Marchenko equations.

The coupled Marchenko equations can be discretized and expressed in a more compact notation using the simplicity of the matrix notation (van der Neut et al., 2015b)

$$
\left[\begin{array}{c}
-\mathbf{g}^{-} \\
\mathbf{g}^{+*}
\end{array}\right]=\left(\mathbf{I}-\left[\begin{array}{cc}
\mathbf{0} & \mathbf{R} \\
\mathbf{R}^{*} & \mathbf{0}
\end{array}\right]\right)\left[\begin{array}{l}
\mathbf{f}^{-} \\
\mathbf{f}^{+}
\end{array}\right],
$$

where $\mathbf{g}^{ \pm}$and $\mathbf{f}^{ \pm}$are vectors in which the seismic traces are concatenated in the time-space domain, whereas $\mathbf{R}$ and $\mathbf{R}^{*}$ can be seen as the multidimensional space-time convolutional and correlational matrix operator, respectively, containing the reflection response acquired. Matrices $\mathbf{I}$ and $\mathbf{0}$ are the identity matrix and a matrix filled with zeros.

\section{Solving the Marchenko equations by LS inversion}

The system of equations (equation 3) represents an underdetermined system of two equations and four unknowns $\left(\mathbf{g}^{+}, \mathbf{g}^{-}, \mathbf{f}^{+}\right.$and $\left.\mathbf{f}^{-}\right)$. To solve the system to get the focusing functions, we resort to the causality properties of the focusing solutions and Green's functions: by noting that the Green's function contains a direct arrival followed by a scattering coda, a window $\Theta$ is designed such that all events after the traveltime $t_{d}\left(\mathbf{x}_{F}, \mathbf{x}_{R}\right)$ of the direct wave from the focal point $\mathbf{x}_{F}$ to the receiver $\mathbf{x}_{R}$ (including the direct wave itself) and before $-t_{d}\left(\mathbf{x}_{F}, \mathbf{x}_{R}\right)$ are zeroed (i.e., $\Theta \mathbf{g}^{+}=\mathbf{0}$ and $\left.\Theta \mathbf{g}^{-}=\mathbf{0}\right)$. On the other hand, the application of the separation operator on the focusing functions leads to $\Theta \mathbf{f}^{+}=\mathbf{f}_{m}^{+}$and $\Theta \mathbf{f}^{-}=\mathbf{f}^{-}$, where we have assumed that $\mathbf{f}^{+}$is composed of a first arrival/direct wave $\mathbf{f}_{d}^{+}$followed by a scattering coda $\mathbf{f}_{m}^{+}$in such a way that $\mathbf{f}^{+}=\mathbf{f}_{d}^{+}+\mathbf{f}_{m}^{+}$ (Wapenaar et al., 2014).

Hence, applying the window matrix $\Theta$ to equation 3 , it is now possible to solve the coupled Marchenko equations for the focussing functions by solving the following system

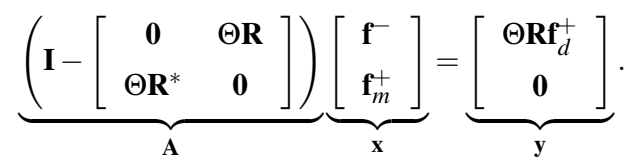

Now, we have that the system of equations given by equation 4, represents a linear system $\mathbf{A x}=\mathbf{y}$, which can be solved by calculating the least-squares (LS) inverse of the matrix A. Another way to solve equation 4 is using the Neumann series to expand the inverse of the matrix $\mathbf{A}$, once this equation is a discretized Fredholm integral equation of the second kind (Slob et al., 2014). Solving equation 4 allows us to obtain $\mathbf{f}^{+}$and $\mathbf{f}^{-}$, which are substituted into equation 3 to compute Green's function components at the desired subsurface point and after that, apply an imaging condition. To construct upand downgoing focusing functions and sub-sequently the Green's function, equation 4 is inverted using an iterative damped LSQR gradient-based optimization scheme (Paige and Saunders, 1982), which minimize a regularized $\ell^{2}$ normal misfit function (e.g., $\min _{\mathbf{x}}\|\mathbf{A x}-\mathbf{y}\|^{2}+\lambda\|\mathbf{x}\|^{2}$ ), and for a consistent set of equations will converge to a solution with a monotonically decreasing residual. The parameter $\lambda$ is a damping factor which was set to $10^{-2}$. In this method, the elements of $\mathbf{A}$ are applied as operators, so it is not necessary to build the referred matrix. The LS scheme is not based on the Neumann expansion series and so it does not require to satisfy a stability criterion.

\section{On the imaging condition}

For any migration algorithm, the construction of a seismic image requires the application of an imaging condition. In possession of the unidirectional components of the Green's function, retrieved by the LS scheme, the imaging principle for the Marchenko imaging consists in applying multidimensional deconvolution (MDD, Wapenaar et al. (2008)). Since the application of this image condition is very expensive, Behura et al. (2014) suggest applying the conventional cross-correlation between these components of Green's functions. However, this imaging condition still contains artifacts because the $g^{+}$component consists of additional events apart from the first arrival. According to van der Neut et al. (2018) and Matias et al. (2018), these artifacts caused by the interaction of multiples in $g^{+}$and primaries (plus multiples) in $g^{-}$, can be avoided by removing the multiples in $g^{+}$, but not by removing the multiples in $g^{-}$. Therefore, substituting $g^{+}$by the initial focusing function, the imaging condition, in the frequency domain, is expressed as

$$
I\left(\mathbf{x}_{I}\right)=\sum_{\mathbf{x}_{S}} \sum_{\omega} g^{-}\left(\mathbf{x}_{I}, \mathbf{x}_{S}\right) f_{d}^{+}\left(\mathbf{x}_{I}, \mathbf{x}_{S}\right),
$$

where $\mathbf{x}_{I}$ and $\mathbf{x}_{S}$ represent the focal point coordinates in the subsurface and the sources/receivers position at the acquisition surface, respectively.

\section{LS - Marchenko imaging in Julia programming}

Julia was chosen for this implementation, even though it is a young general-purpose programming language, which has proven to be very efficient and with easy syntax for code implementation (Gao et al., 2020). The used version was Julia 1.5.3. As mentioned, the recovery of the up- and downgoing focusing functions will be carried out through the LS scheme applied to equation 4, and for this the LSQR algorithm proposed by Paige and Saunders (1982) will be used. This inversion scheme is implemented in various programming languages. For Julia, the code corresponding to the LSQR scheme is inside the package IterativeSolvers, which provides iterative algorithms for solving linear systems, eigensystems, and singular value 
problems.

For the implementation of the LS solution of the Marchenko coupled equations system, the Isqr function will be used. Usually, this function has as input parameters the matrix $\mathbf{A}$ and the column vectors $\mathbf{y}$ and $\mathbf{x}$, where $\mathbf{x}$ can be a column vector filled with zeros. In addition, in the $l s q r$ function the matrix-vector product is used in a recurrent way to update $\mathbf{x}$ and $\mathbf{y}$ in the next iteration, a product that can be expressed as follows

$$
\begin{aligned}
& \mathbf{y} \leftarrow \mathbf{y}+\mathbf{A x} \\
& \mathbf{x} \leftarrow \mathbf{x}+\mathbf{A}^{T} \mathbf{y} .
\end{aligned}
$$

For the LS approach of the Marchenko equations, we have the matrix $\mathbf{A}$ composed of a set of sub-matrices, as shown in equation 4 . So the basic idea here is creating a function called APROD (see Algorithm 1) that lets us choose when to do the operations $\mathbf{A} \mathbf{x}$ and $\mathbf{A}^{T} \mathbf{y}$, where

$$
\mathbf{A}=\left[\begin{array}{cc}
\mathbf{I} & -\Theta \mathbf{R} \\
-\Theta \mathbf{R}^{*} & \mathbf{I}
\end{array}\right] \text { and } \mathbf{A}^{T}=\left[\begin{array}{cc}
\mathbf{I} & -\Theta \mathbf{R}^{*} \\
-\Theta \mathbf{R} & \mathbf{I}
\end{array}\right]
$$

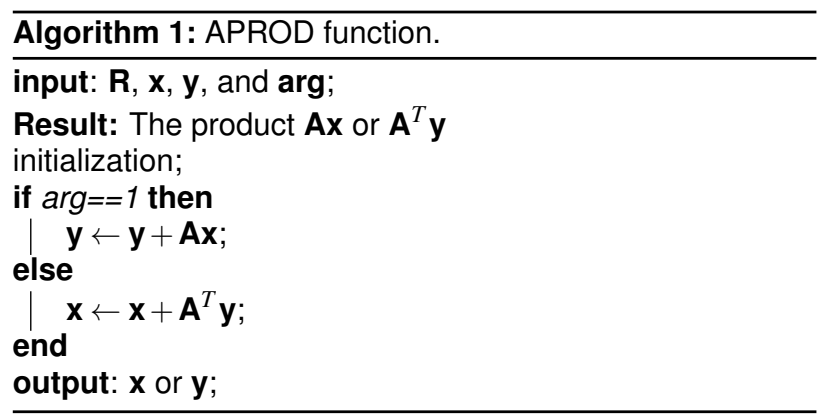

Finally, with the recovery of unidirectional components of Green's function, it is possible to apply the crosscorrelation image condition given by equation 5 and generate the migrated section. The pseudocode for the Marchenko imaging, implemented in this work, is given by Algorithm 2.

Algorithm 2: Marchenko imaging algorithm. The notation used in the pseudocode is the same notation from Marchenko equations section.

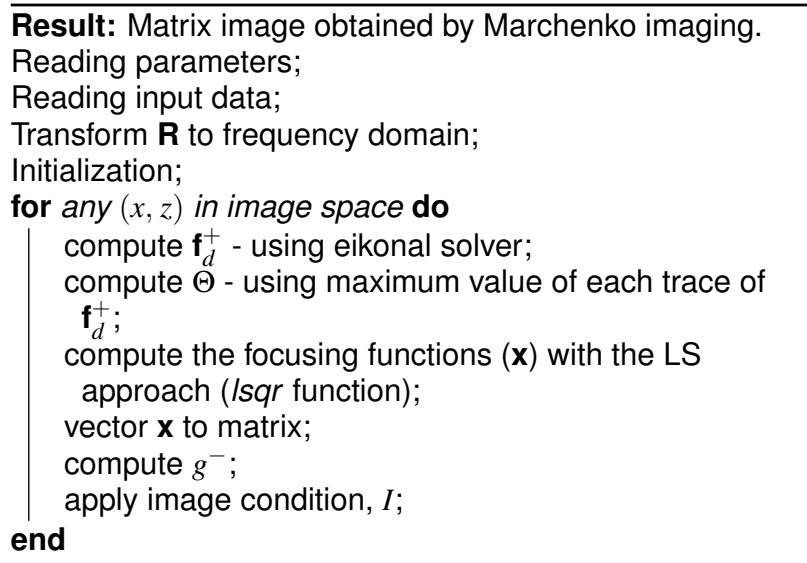

\section{NUMERICAL EXAMPLE}

In the following, we use a $2 \mathrm{D}$ synthetic model to illustrate the Marchenko algorithm and its benefits to generating seismic images. The reflection response are modeled in a constant-velocity $(c=2400 \mathrm{~m} / \mathrm{s})$, variable-density model. Figure 1 shows the values for the density as a function of depth and horizontal position. The density model is composed of flat layers and a syncline structure. Density values in the upper part of the model expose very strong contrasts, so this model will be able to generate the interbed reflections needed for our tests. The model consists of $1201 \times 641$ grid nodes with $2.5 \mathrm{~m}$ grid spacing. We have simulated the synthetic acoustic impulse reflection responses $R$ using a finite-difference time-domain modeling code (Thorbecke and Draganov, 2011), and the input source signature is approximately a sinc function with a flat spectrum of unitary amplitude. We computed the single-sided reflection responses with 201 sources and a fixed-spread geometry that ranges from -1500 to $1500 \mathrm{~m}$ with a $15 \mathrm{~m}$ distance between sources and also between receivers, which are located at the acquisition surface the top of the model. The duration of each shot record is $1.5 \mathrm{~s}$ sampled at $2 \mathrm{~ms}$. Absorbing boundary conditions are applied on all sides, i.e., we assume that surface-related multiples and ghost wave effects are removed from the recorded dataset. The direct wave has been removed from the modeled reflection responses by modeling it separately in a homogeneous medium (values of the first layer) and then subtracting it from the recorded data. The central shot (orange star in Figure 1) of the computed single-sided reflection responses $R$ is shown in Figure 2a, where we can see the presence of internal multiple reflections.

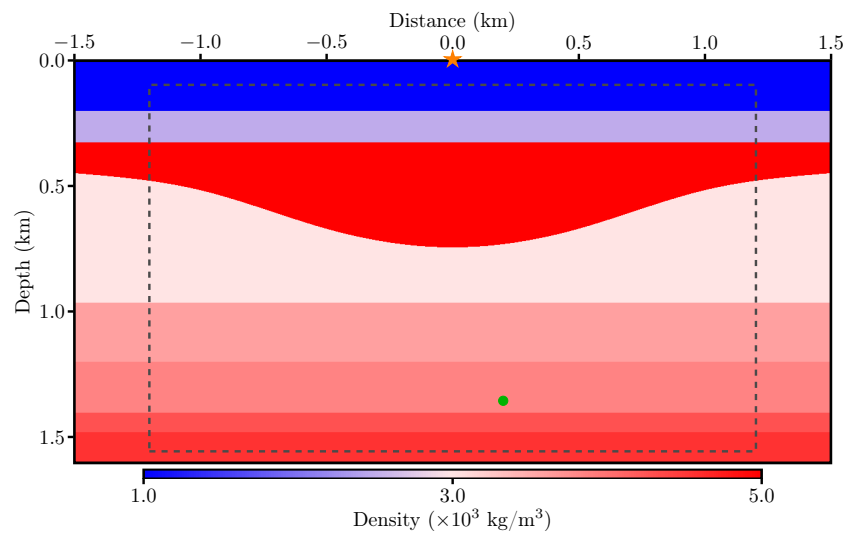

Figure 1: Constant velocity, variable density subsurface model used to generate the single-sided reflection response. The green dot indicates the subsurface focal point where the Green's function is computed and shown in Figure 3. The dashed gray line indicates the target area for imaging.

The first arrival of the downgoing Green's function, recorded at the surface acquisition for a source at $\mathbf{x}=$ $(200,1350) \mathrm{m}$ (green dot in Figure 1), is shown in Figure 2b. It was constructed by the convolution of the traveltimes computed from the eikonal equation solver (Faria and Stoffa, 1994) and a zero-phase Ricker source wavelet that has its central frequency at $20 \mathrm{~Hz}$. The modeled single-sided reflection responses and time-reversed first arrivals are used as inputs to construct up- and downgoing 
focusing functions and subsequently the Green's function, and for this, equation 4 is inverted using the LS scheme. The number of iterations for the LS technique is set at 20. In Figure 3, the Marchenko-computed Green's function is compared to the reference Green's function, which was modeled using finite differences. The Green's function recovered by the Marchenko scheme presents events similar to those contained in the reference function. However, there are some differences in the amplitudes of these events. The colored lines in Figure $3 a-b$ represent the zero-offset trace positions that were selected for a more detailed analysis. All traces in Figure $3 c$ have been normalized by their maximum amplitude. In Figure $3 \mathrm{c}$ we perform a comparison between these traces, and we can see that the Marchenko scheme successfully retrieves the Green's function, thus preserving their phase. According to Thorbecke et al. (2017), the small amplitude mismatch present at earlier times is related to the method employed to estimate the direct arrival, while that, after approximately $0.8 \mathrm{~s}$, the amplitude error decreases because the presence of higher wavenumbers becomes smaller.
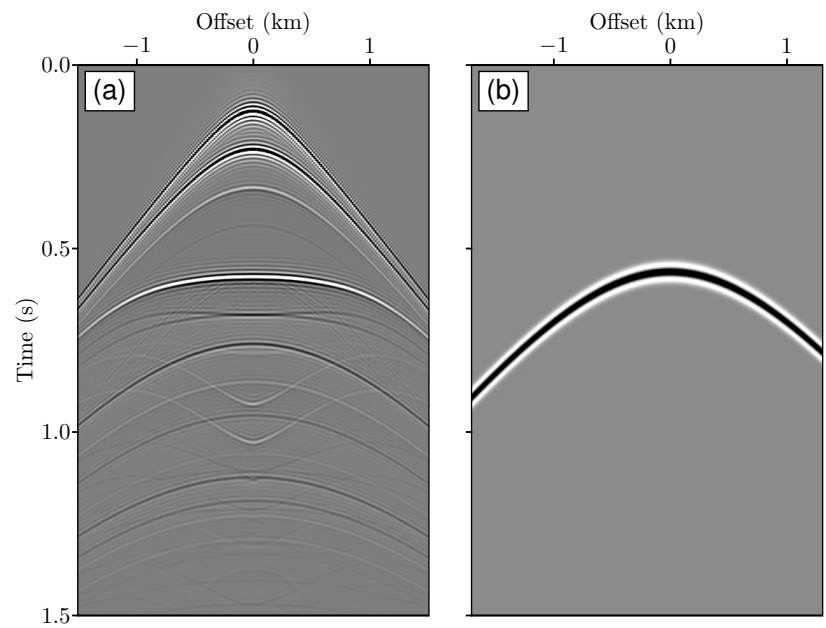

Figure 2: Common shot gather with source position at $\mathbf{x}=(0,0) \mathrm{m}$ (orange star in Figure 1) and receivers at the acquisition surface. (b) The modeled first arrival of the downgoing Green's function from a source at $\mathbf{x}=$ $(200,1350) \mathrm{m}$ (green dot in Figure 1) and receivers at the acquisition surface. Note that the data on the left are a broadband data (i.e., flat spectrum wavelet), whereas the first arrival on the right is shaped by a $20 \mathrm{~Hz}$ Ricker wavelet.

In the following, we apply the Marchenko imaging scheme for the zone limited by the gray dashed line in Figure 1 , where the selected focal points cover an area of 241 by 146 points $(-1200 \mathrm{~m} \leq x \leq 1200 \mathrm{~m}$ and $100 \mathrm{~m} \leq z \leq$ $1550 \mathrm{~m}$, a total of 35186 points imaged). To generate a reference image, we have applied the imaging condition (equation 5) using the upgoing component of the retrieved Green's function at the first iteration of the LSQR scheme, which leads to the conventional RTM image of the target zone (Zhang et al., 2018). A comparison between the migrated images is shown in Figure 4. The image in Figure 4a contains artifacts (indicated by the red arrows) from internal multiple reflections because they are imaged as if they were primary reflections. However, the image in Figure $4 \mathrm{~b}$, which is obtained by the Marchenko imaging, is nearly perfect without ghost images due to internal multiple reflections. We should notice that this result has a better quality compared to Figure 4a. These results confirm the successful application of the Marchenko imaging and show the effectiveness of the implemented algorithm for attenuating the harmful ghost reflections usually seen in a conventional RTM.
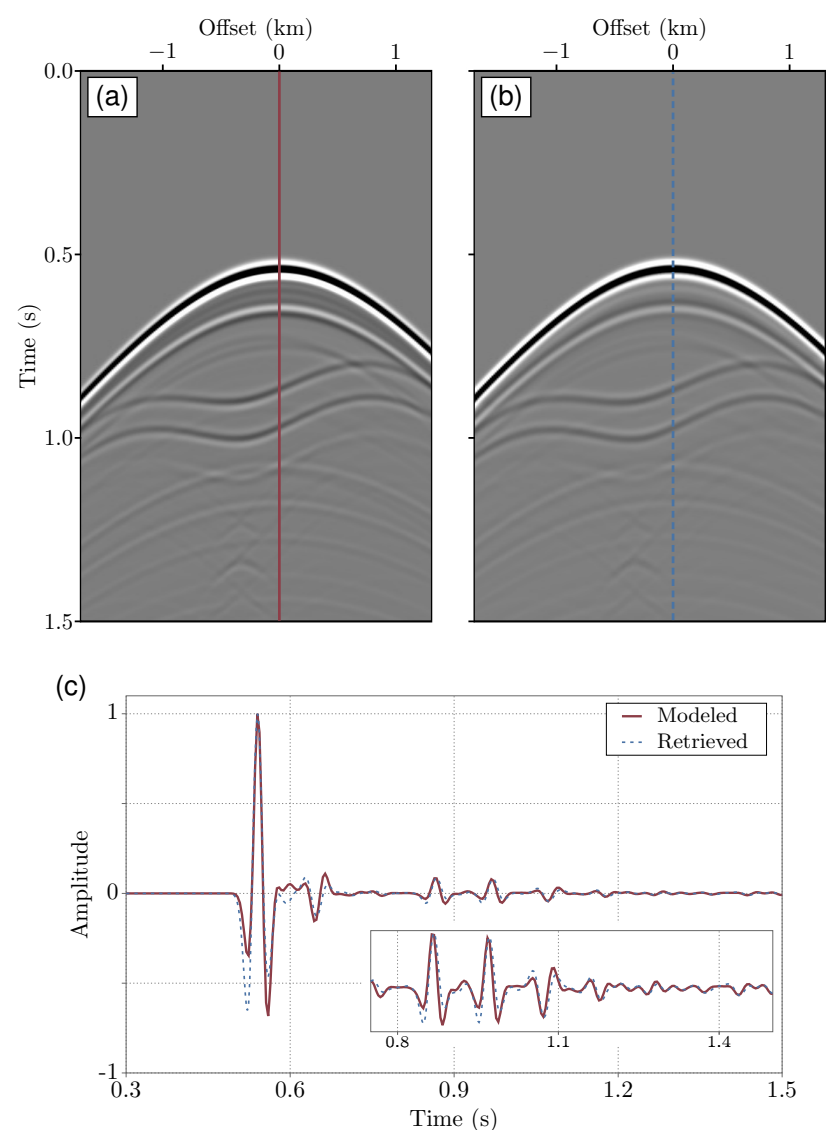

Figure 3: (a) The reference Green's function and (b) the Green's function computed with the Marchenko method after twenty iterations. (c) The comparison of zero-offset traces.

From the numerical results, we test and also show the functionality of our implementation of the Marchenko method using the Julia programming language. The libraries used here were really useful and helpful to save o lot of time in the implementation. Besides, Julia allows us the possibility to use another language (e.g., python, Fortran and $\mathrm{C} / \mathrm{C}++)$, which brings versatility to the language and makes it possible to use a lot of readymade routines. For future works, the comparison between the iterative substitution and the LS scheme, in Julia, can be done, and we also suggest the use of PyLops from Python to see how this library can speed up the current implemented code.

\section{CONCLUSIONS}

The Julia programming language was efficient in geophysical terms to compute the Green's functions and the migrated image using the Marchenko method. In the numerical examples, the comparison between the first and the iteration twenty shows that many of artifacts were attenuated, demonstrating the functionality of the 
code. Pre-existing libraries with the high-level language that Julia offers accelerate the implementation of the Marchenko algorithm with the LS approach. The Julia code could benefit from further optimizations, especially when considering high size seismic data sets.
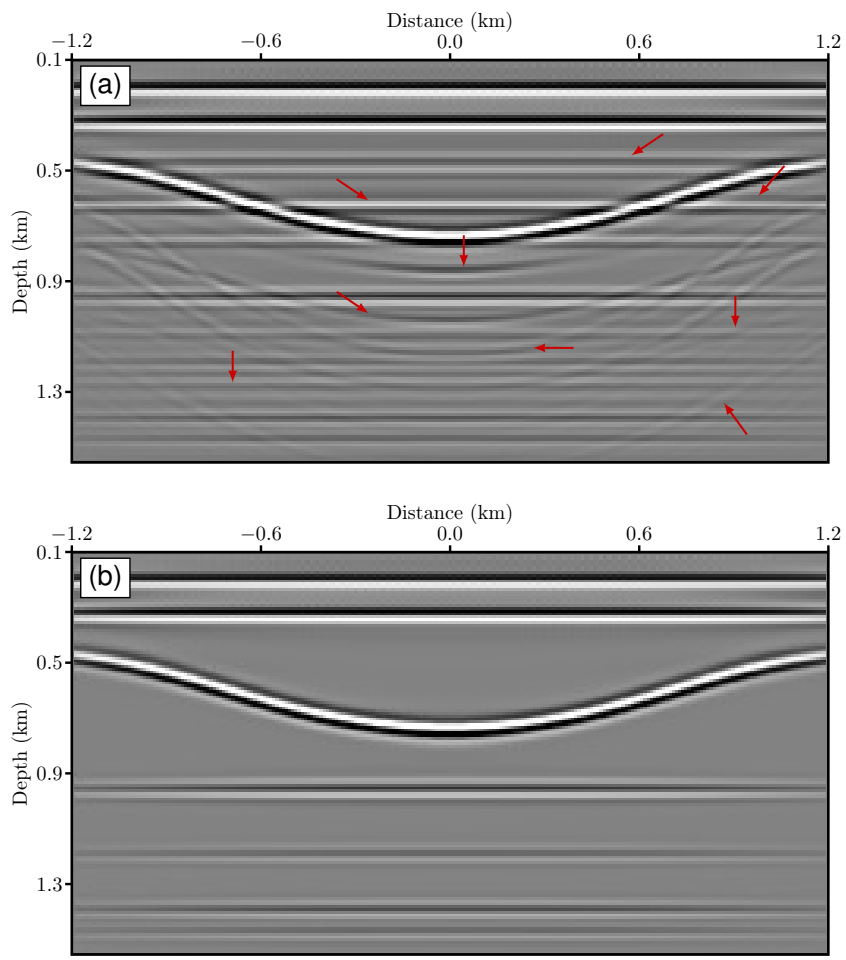

Figure 4: (a) The image retrieved from the modeled reflection response and (b) the artifact-free image of the target zone retrieved by the Marchenko imaging. The red arrows in (a) indicate artifacts arising from internal multiple reflections.

\section{ACKNOWLEDGMENTS}

This work was supported by CENPES/Petrobras through the Marchenko project at SENAI-CIMATEC. The authors also wish to thank to the developer team of the Open-Source libraries in TUDelft, and a special acknowledgment to the geophysical team at SENAICIMATEC for discussions and contributions.

\section{References}

Baysal, E., D. D. Kosloff, and J. W. Sherwood, 1983, Reverse-time migration: Geophysics, 48, no. 11, 15141524.

Behura, J., K. Wapenaar, and R. Snieder, 2014, Autofocus imaging: image reconstruction based on inverse scattering theory: Geophysics, 79, A19-A26.

Bezanson, J., S. Karpinski, V. B. Shah, and A. Edelman, 2012, Julia: A fast dynamic language for technical computing: arXiv preprint 1209.5145.

Broggini, F. and R. Snieder, 2012, Connection of scattering principles: a visual and mathematical tour: European Journal of Physics, 33, 593-163.

de Hoop, A. T., 1995, Handbook of Radiation and Scattering of Waves: Academic Press.

Dukalski, M. and K. de Vos, 2018, Marchenko inversion in a strong scattering regime including surface-related multiples: Geophysical Journal International, 212, no. 2, 760-776.

Faria, E. L. and P. L. Stoffa, 1994, Traveltime computation in transversely isotropic media: Geophysics, 59, 272-281.

Gao, K., G. Mei, F. Piccialli, S. Cuomo, J. Tu, and Z. Huo, 2020, Julia language in machine learning: Algorithms, applications, and open issues: Computer Science Review, 37, 100254.

Gevorkyan, M., A. Demidova, A. Korolkova, and D. Kulyabov, 2019, Statistically significant performance testing of Julia scientific programming language: Journal of Physics: Conference Series, 12-17.

Hokstad, K., 2000, Multicomponent Kirchhoff migration: Geophysics, 65, 861-873.

Matias, M. M. A., R. C. Pestana, and J. van der Neut, 2018, Marchenko imaging by unidimensional deconvolution: Geophysical Prospecting, 66, 1653-1666.

Paige, C. and M. Saunders, 1982, Algorithm 583. LSQR: Sparse linear equations and least squares problems: ACM Transactions on Mathematical Software, 8, 195209.

Ravasi, M., 2017, Rayleigh-Marchenko redatuming for target-oriented, true-amplitude imaging: Geophysics, 82, no. 6, S439-S452.

Ravasi, M. and I. Vasconcelos, 2020a, Implementation of Large-Scale Integral Operators with Modern HPC Solutions: 2020, 1-5.

- 2 2020b, PyLops - A linear-operator Python library for scalable algebra and optimization: SoftwareX, 11, 100361.

Slob, E., K. Wapenaar, F. Broggini, and R. Snieder, 2014, Seismic reflector imaging using internal multiples with marchenko-type equations: Geophysics, 79, S63-S76.

Thorbecke, J. and D. Draganov, 2011, Finite-difference modeling experiments for seismic interferometry: Geophysics, 76, no. 6, H1-H18.

Thorbecke, J., E. Slob, J. Brackenhoff, J. van der Neut, and K. Wapenaar, 2017, Implementation of the Marchenko method: Geophysics, 82, no. 6, WB29-WB45.

van der Neut, J., J. Brackenhoff, M. Staring, L. Zhang, S. de Ridder, E. Slob, and K. Wapenaar, 2018, Singleand Double-Sided Marchenko Imaging Conditions in Acoustic Media: IEEE Transactions on Computational Imaging, 4, no. 1, 160-171.

van der Neut, J., J. Thorbecke, K. Wapenaar, and E. Slob, 2015a, Inversion of the multidimensional Marchenko equation: 77th EAGE Conference and Exhibition 2015, $1-5$.

van der Neut, J., I. Vasconcelos, and K. Wapenaar, 2015b, On Green's function retrieval by iterative substitution of the coupled Marchenko equations: Geophysical Journal International, 203, 792-813.

van der Neut, J., K. Wapenaar, J. Thorbecke, E. Slob, and I. Vasconcelos, 2015c, An illustration of adaptive Marchenko imaging: The Leading Edge, 34, 818-822.

Vargas, D., I. Vasconcelos, Y. Sripanich, and M. Ravasi, 2021, Scattering-based focusing for imaging in highly complex media from band-limited, multi-component data: Geophysics, 86, 1-64.

Wapenaar, C. P. A. and J. L. T. Grimberg, 1996, Reciprocity theorems for one-way wave fields: Geophysical Journal International, 127, 169-177.

Wapenaar, K., D. Draganov, and J. O. A. Robertsson, 2008, Redatuming: Seismic Interferometry, Geophysics Reprint Series, 331-448. 
Wapenaar, K., J. Thorbecke, J. van der Neut, F. Broggini, E. Slob, and R. Snieder, 2014, Marchenko imaging: Geophysics, 79, no. 3, WA39-WA57.

Zhang, L., E. Slob, J. van der Neut, and K. Wapenaar, 2018, Artifact-free reverse time migration: Geophysics, 83, no. 5, A65-A68. 